\title{
Immuno-detection of dioxins using a recombinant protein of aryl hydrocarbon receptor (AhR) fused with sfGFP
}

\author{
Walaa Faiad ${ }^{1}$, Abdulsamie Hanano², Mohamed Maher Kabakibi ${ }^{1}$ and Abdul Qader Abbady ${ }^{3^{*}}$ (D)
}

\begin{abstract}
Background: Dioxins are one of the most toxic groups of persistent organic pollutants. Their bioaccumulation through the food chain constitutes a potential risk for human health. Upon cell entry, dioxins bind specifically and firmly to the aryl hydrocarbon receptor (AhR), leading to the stimulation of several enzymes responsible for its detoxification. Dioxin/AhR interaction could be exploited as an affordable alternative to a variety of analytical methods for detecting dioxin contamination in the environment.

Results: In this work, the ligand binding domain (LBD) of the AhR was cloned downstream a superfolder form of the green fluorescent protein (sfGFP), resulting in the construct pRSET-sfGFP-AhR. High level of expressed sfGFP-AhR fusion protein $(50 \mathrm{kDa})$ was recovered from the inclusion bodies of $E$. coli by simple solubilization with the Arginine, and purified by affinity chromatography via its $\mathrm{N}$-terminal $6 \times$ His tag. Its purity was confirmed by SDS-PAGE analysis and immunoblotting with anti-His or anti-GFP antibodies. Indirect ELISA revealed the ability of the sfGFP-AhR, but not the $s f G F P$, to bind to the immobilized dioxin with the possibility to detect such interaction by both its $6 \times$ His and GFP tags,Competitive ELISA showed that anti-dioxin antibody was more sensitive to low dioxin concentrations than sfGFP-AhR. Nevertheless, the detection range of sfGFP-AhR fusion was much wider and the detection limit was of about 10 ppt (parts per trillion) of free dioxin in the tested artificial samples.
\end{abstract}

Conclusions: this highly expressed and functional sfGFP-AhR fusion protein provides a promising molecular tool for detecting and quantifying different congeners of dioxins.

Keywords: TCDD, sfGFP, AhR, Protein expression, Fusion proteins, Cloning

\section{Background}

Collectively termed as dioxins, polychlorinated dibenzop-dioxins (PCDDs) and polychlorinated dibenzofurans (PCDFs) are the most toxic group of the persistent organic pollutants (POPs). Depending on the chlorination level $(\mathrm{P}=1-8)$, dioxins form a generic group of 75 PCDD congeners and 135 PCDF congeners with varying degrees of toxicity. The congeners with chlorine atoms substituted in the lateral 2, 3, 7 and 8 positions of the aromatic rings are considered as the most toxic. 2,3,7,8-Tetrachlorodibenzo- $p$-dioxin (TCDD), with a toxic equivalency factor (TEF) of 1.0, is the most toxic

\footnotetext{
* Correspondence: aabady@aec.org.sy

${ }^{3}$ Division of Microbiology and Immunology, Department of Molecular Biology and Biotechnology, Atomic Energy Commission of Syria (AECS), Damascus, Syria

Full list of author information is available at the end of the article
}

congener of dioxins [1]. Consequently, TCDD was used as a good candidate for investigations of the physiological and toxicological effects of this class of chemicals $[2,3]$.

Dioxins are a major product of industrial, municipal and domestic incineration and combustion processes [4] and during industrial processes involving chlorinated aromatic and aliphatic compounds, such as pesticides and herbicides synthesis [5]. Currently, incineration of the solid wastes contributes the most to the release of these compounds into the environment [6].

Due to their persistence and high lipophilicity, PCDD/Fs are subject to lipophilic bio-concentration and accumulation in the food chain [7]. Their adverse health effects are now well reported; for example the wasting syndrome [2], immunotoxicity [8], teratogenicity [3], dysfunctional immune and reproductive systems [9] and carcinogenesis 
[10]. An effective and serious contamination with dioxins has been previously reported in soils, sediments, air and water [11, 12]. In this context, numerous analytical techniques have been developed for the detection and quantification of dioxins. High resolution gas chromatography combined with high resolution mass spectrometry (HRGC/HRMS) is considered the most reliable and sensitive method for separation, identification and determination of the congener-specific concentrations of dioxins [13]. However, this technique requires a sophisticated platform and highly trained personnel, besides being very costly and time-consuming. Consequently several rapid and inexpensive screening assays have been proposed. Immuno-detection, which depends on the ability of a certain compound to be precisely recognized and bound by a specific antibody, makes the base of what is known as the enzyme-linked immunosorbent assay (ELISA) [14, 15]. Alternatively, the detection of signal transduction effects that are related to dioxin stimulation in vitro or in cells has been exploited using sophisticated methods; such as the chemical activated luciferase gene expression (CALUX) system [13].

The biological effects of the dioxins in the body critically depend on a cytosolic protein, the aryl hydrocarbon receptor (AhR) [16]. The AhR resembles nuclear receptors (among which it was initially categorized) acting as a ligand-activated transcription factor [16]. In response to activation by dioxins, AhR signalling pathway modifies the expression levels of numerous genes. The best characterized of these pathways at the molecular level is that leading to the induction of the gene for a Phase I drug-metabolizing enzyme; CYP1A1 [17]. In the dormant state, the AhR resides in the cytosol in a protein complex also containing a dimer of heat shock protein 90 (HSP90), an AhR-associated protein-9 and p23 [18]. These chaperone proteins stabilize the AhR maintaining it in a conformation that is unable to enter the nucleus but optimal for ligand binding [19]. The AhR then dissociates from the chaperones and heterodimerizes with the AhR nuclear translocator (ARNT) [20]. AhR/ARNT dimer binds to the major groove of the DNA helix at specific sites [21]. Structurally, the AhR belongs to basic Helix-Loop-Helix/Periodic, AhR nuclear translocator, single-minded (bHLH/PAS) proteins. The AhR contains several important structural elements; including an $\mathrm{N}$-terminal bHLH domain, a C-terminal transcriptional activation domain and a central PAS domain containing two degenerated repeats (PAS-A and PAS-B). The ligand binding domain (LBD) of the AhR is localized between amino acids 230 and 397 [22], and has a similar affinity for TCDD binding as the full-length AhR [23].

In line of these insights we investigated the possibility to fuse the LBD of the AhR with a marker protein, the green fluorescent protein (GFP), in order to exploit the resulting recombinant fusion protein in dioxins detection. The GFP from Aequorea jellyfish is widely used as an excellent expression tag for several fusion proteins [24], and it has been expressed in a variety of species; including bacteria, plants, Drosophila melanogaster, Caenorhabditis elegans, zebrafish, andmammals [24-26]. Waldo and co-workers reported the engineering of an enhanced superfolder $(s f)$ form of the GFP, and this sfGFP showed increased resistance to denaturation, improved folding kinetics, and increased resistance to aggregation during protein expression [27]. Therefore, the main objective of the current study was to express the $s f$ GFP-AhR fusion protein in E. coli. Computational 3D structure prediction of this fusion suggested a total accessibility of the AhR moiety for TCDD binding, and consequently this was confirmed in our immunological experiments. Optimized $s f$ GFP-AhR fusion could effectively be invested in as an affordable, rapid, sensitive and environment-friendly technique to detect the presence of dioxins in environment samples and food commodities.

\section{Methods \\ Preparation of the AhR cDNA from HepG2 cell line}

Total RNA was extracted from the human hepatoma cells (HepG2) using IllustraRNAspin Mini Kit (GE Life Sciences) according to the manufacturer's manual. $2 \mu \mathrm{g}$ of the RNA was reverse-transcribed to the cDNA using Ready-to-Go You-prime first-strand-beads (GE Life Sciences) with oligo-d $\mathrm{T}^{15-18}$ (Invitrogen). $2 \mu \mathrm{l}$ of the cDNA was used as a template in a PCR with a pair of specific primers for the AhR(LBD); $\mathrm{AhR}(\mathrm{BamHI})-\mathrm{F}$ and AhR(EcoRI)-R (Additional file 1: Table S1). Primers were designed to amplify the LBD of the $A h R$ gene and to add $B a m \mathrm{HI}$ and EcoRI restriction sites at the $5^{\prime}$ and $3^{\prime}$ ends, respectively. The fragment of LBD was amplified by a high fidelity Taq DNA polymerase (AccuPrime ${ }^{\mathrm{Tm}}$ Kit; Invitrogen) at $55{ }^{\circ} \mathrm{C}$ annealing temperature. Amplified AhR fragment and the pRSET-sfGFP plasmid [28] were digested with $B a m H I$ and EcoRI (Fermentas) then ligated using the T4 DNA ligase following standard procedures (Fermentas).

\section{Bacterial strains and growth conditions}

E. coli strains TOP10 (Invitrogen) and BL21(DE3) Gold (Novagen) were used in cloning and protein expression. E. coli was grown in Luria Broth (LB; 1 \% Tryptone, $0.5 \%$ yeast extract, $171 \mathrm{mM} \mathrm{NaCl}$ ) (Bio Basic Inc.) with ampicillin antibiotic $(100 \mu \mathrm{g} / \mathrm{ml}$, Sigma $)$ in orbit-rotating incubator at $37{ }^{\circ} \mathrm{C}$. Freshly prepared electro-competent E. coli TOP10 cells were transformed with the plasmid constructs by electroporation. Colony PCR screening for positive AhR clones was performed using pRSET specific primers $\left(\mathrm{T}_{7} \mathrm{~F} / \mathrm{T}_{7} \mathrm{R}\right)$ (Additional file 1: Table S1). The plasmid constructs were isolated from some positive 
clones by plasmid Miniprep Kit (Qiagen) after being grown in LB/ampicillin medium. The identity of these plasmid constructs was confirmed by digestion with the restriction enzymes and by sequencing using different sequencing primers covering the full-length LBD (Additional file 1: Table S1).

\section{Expression of the sfGFP-AhR fusion protein as inclusion bodies}

The confirmed plasmid constructs; the pRSET-sfGFP and pRSET-sfGFP-AhR, were used to transform E. coli BL21 (DE3) Gold cells by electroporation. Protein expression was performed in $250 \mathrm{ml}$ shake flasks by growing the bacteria in LB medium till an optical density of 0.5 to 0.7 was reached, and then the expression was induced with 0.5 mMIsopropylthio-D- galactoside(IPTG, Promega) for $16 \mathrm{~h}$ at $15{ }^{\circ} \mathrm{C}$. After centrifugation, the pellet of bacteria was resuspended on the binding buffer $(50$ mMtris- $\mathrm{HCl} \mathrm{pH}$ 8.0, $150 \mathrm{mM} \mathrm{NaCl}$ and 1 mMphenylmethylsulfonyl fluoride (PMSF, Sigma) supplemented with a complete protease inhibitor cocktail (Roche), then lysed by 8 pulses ( $15 \mathrm{~s}$ each) of sonication at $22 \%$ amplitude (750-Watt Ultrasonic Homogenizer, Cole-Parmer) in ice. The lysate containing soluble proteins as the $s f$ GFP was cleared by centrifugation at $12000 \times \mathrm{g}$ for $20 \mathrm{~min}$ at $4{ }^{\circ} \mathrm{C}$. The pellet of the inclusion bodies containing insoluble proteins (as the sfGFP-AhR) was resuspended by sonication in the same binding buffer supplemented with (5 mM DTT, 5 mM EDTA, $1 \mathrm{M}$ urea and $1 \%$ triton-x). This washing step is designed to remove contaminants, especially proteins that may have adsorbed onto the hydrophobic inclusion bodies during processing and could affect protein refolding yield. After washing with the basic binding buffer to eliminate the detergents, inclusion bodies were ready to be solubilized using the different reagents.

\section{Refolding and solubilizion of sfGFP-AhR inclusion bodies}

To assess the optimal reagent for assisting the correct refolding of the sfGFP-AhR, inclusion bodies were prepared at $0.1 \mathrm{mg} / \mathrm{ml}$ TBS buffer $(20 \mathrm{mMtris}-\mathrm{HCl} \mathrm{pH} 8.0$ and $150 \mathrm{mM} \mathrm{NaCl}$ ). $500 \mu \mathrm{l}$ of this preparation was distributed into several $1.5 \mathrm{ml}$ microtubes then centrifuged at max speed for $5 \mathrm{~min}$. The pellets in the microtubes were dispersed and dissolved by sonication in the same buffer containing different concentration of various additives reported in the literature [29] (Additional file 2: Table S2). After 30 min of incubation at RT, supernatant (soluble fraction) was recovered from each condition by centrifugation and the remaining pellet of insoluble sfGFP-AhR was dissolved by sonication in $500 \mu \mathrm{l}$ of $8 \mathrm{M}$ urea-containing buffer (insoluble fraction). The formation of a fluorophore in the soluble and insoluble fractions was monitored by measuring the fluorescence density, expressed as a relative fluorescent unit (RFU), at excitation wavelength $485 \mathrm{~nm}$ with emission wavelength $538 \mathrm{~nm}$ (Fluoroskan Ascent, Thermo Labsystems), and the fluorophore fluorescein (Sigma) was used as a control.

\section{Purification of the sfGFP and sfGFP-AhR fusion proteins} Soluble fractions of $E$. coli extract containing the sfGFP and Arginine-refolded sfGFP-AhR were dialysed in binding buffer containing $20 \mathrm{mM}$ imidazole and then purified using a $5 \mathrm{ml}$ column of nickel charged nitrilotriacetic acid (NTA) superflow sepharose (Qiagen) installed on the fast protein liquid chromatography (FPLC) AKTA prime plus system (GE Life Sciences). After washing, bound proteins were eluted from the column with $500 \mathrm{mM}$ imidazolecontaining buffer. The eluted fraction was concentrated on Vivaspin concentrators with a molecular mass cut-off of $10 \mathrm{kDa}$ (Vivascience). The concentration of the purified proteins was determined by the Bradford method, and their purity was evaluated in SDS-PAGE prepared using stacking gel $5 \%$ and running gel $12 \%$. After electrophoresis, the gel was stained in coomassie brilliant blue buffer (45\% methanol, $10 \%$ acetic acid and $0.25 \%$ coomassie R250) for $2 \mathrm{~h}$ and then washed several times in the destaining buffer.

\section{Immunoblotting of the sfGFP and sfGFP-AhR}

Purified proteins were separated by SDS-PAGE before blotting onto $0.45 \mu \mathrm{m}$ nitrocellulose membranes (BioRad) using $1 \times$ transfer buffer ( $25 \mathrm{mMtris}-\mathrm{HCl}, 200 \mathrm{mM}$ glycine, $0.1 \%$ SDS and $20 \%$ methanol). After blotting, the membranes were washed with TBS-T $(0.05 \%$ Tween-20 in TBS) then incubated in the blocking buffer (5\% skimmed milk in TBS-T). The membranes were incubated with 1:2000 dilution of either mouse anti-GFP (Roche) or mouse anti- $6 \times$ His (R\&D Systems) antibodies for $1 \mathrm{~h}$ at RT. After several washes with TBS-T, the membranes were incubated with (1:2000) dilution of a secondary goat anti-mouse antibody conjugated to the horseradish peroxidase (HRP, Bethyl laboratories) for $1 \mathrm{~h}$ at RT. Bands revelation was achieved by adding 3-amino9-ethylcarbazole (AEC, Sigma) chromogen substrate in acetate buffer in the presence of a hydrogen peroxide.

\section{Indirect ELISA assay for testing sfGFP-AhR}

An indirect ELISA was performed by immobilizing the antigens $(10 \mathrm{nM}), s f G F P-A h R$ or $s f G F P$, on a standard MaxiSorp 96-well plate (Nunc) overnight in a bicarbonate/ carbonate coating buffer $(100 \mathrm{mM}, \mathrm{pH}$ 9.6). Alternatively, dioxin binding was tested using a TCDD-pre-coated 96well ELISA microplate (Abraxis LLC). Before use, all different plates were incubated in the blocking buffer $(5 \%$ skimmed milk in TBS-T) for $2 \mathrm{~h}$ at RT then washed 3 times with TBS-T. Immobilized TCDD in the wells was directly detected using a rabbit-anti-TCDD (Abraxis), 
following the manufacturer's instructions. Otherwise, the $s f$ GFP-AhR and sfGFP were diluted to the desired concentration $(100 \mathrm{nM})$ in $1 \%$ blocking buffer then applied to the wells $(100 \mu \mathrm{l})$ for $16 \mathrm{~h}$ at $4{ }^{\circ} \mathrm{C}$, then washed 3 times with TBS-T. Antigens (immobilized or bound to TCDD) were detected by an incubation for $1 \mathrm{~h}$ at RT with a diluted (1:2000) mouse anti- $6 \times$ His, rabbit anti- $6 \times$ His (Bethyl laboratories), mouse anti-GFP or rabbit anti-GFP (a homemade product, [28]). After 5 washes, diluted (1:2000) goat anti-mouse or goat-anti-rabbit IgGs conjugated to horseradish peroxidase (HRP, Bethyl laboratories) were added to the appropriate wells. The wells were then washed 5 times before bound conjugates were detected with $50 \mu \mathrm{l}$ of 3,3',5,5' -tetramethylbenzidine (TMB, Sigma) ready-to-use substrate. The reaction was finally stopped with the addition of $50 \mu \mathrm{l}$ of $1 \mathrm{M} \mathrm{H}_{2} \mathrm{SO}_{4}$. The spectroscopic absorbance of each well was measured in an automated plate reader at a wavelength of $450 \mathrm{~nm}$.

\section{TCDD detection by a competitive indirect ELISA using the sfGFP-AhR}

Using the same TCDD-pre-coated plates and doing the same primary steps of blocking and washing, the competitive ELISA was carried out by incubating the sfGFPAhR (30 nM) or rabbit-anti-TCDD with serial dilutions (from 0 - to 100,000 parts per trillion (ppt) in $1 \%$ DMSO-containing blocking buffer) of free TCDD (stock at $10 \mu \mathrm{g} / \mathrm{ml}$, purity $99 \%$, Supelco Inc.) in $100 \mu \mathrm{l}$ volume for $2 \mathrm{~h}$ at RT. Different conditions were then transferred to TCDD-pre-coated wells and incubated for further $2 \mathrm{~h}$ at RT. The following steps were performed as described earlier using a rabbit-anti-GFP antibody, goat-antirabbit-HRP and the TMB substrate. The experiments were performed three times to determine the mean values (used to draw the graphs) and the error bars were calculated by dividing the standard deviation of the data by the square root of number (3) of values that make up the mean.

\section{Results}

\section{Design of the sfGFP-AhR fusion protein}

Human $A h R$ is a relatively large protein of $96 \mathrm{kDa}$ composed of 848 amino acids (aa), extending over several well identified functional domains (Fig. 1a). LBD positioned from 231 to 428 was cloned downstream of the $s f$ GFP gene in the plasmid pRSET-sfGFP, resulting in a fusion protein of $50 \mathrm{kDa}$ (Fig. 1a). The resulting sfGFP-AhR fusion protein contains an N-terminal $6 \times$ His-tag and a free and flexible LBD moiety exposed an internal polypeptide linker GGSSSG at its C-terminal (Fig. 1a). A soluble cytoplasmic N-terminal $6 \times$ His-tagged $s f G F P$ of $27 \mathrm{kDa}$ was also expressed in E. coli using the original plasmid pRSET-sfGFP (Fig. 1a) [28, 30]. A simple 3D-modeling of the cloned LBD showed the cavity for TCDD binding that extends from 284 to 390 aa, overlapping entirely with the PAS-B domain and containing several aa whose side chains are critical for TCDD binding. These aa are found to be highly conserved between human, mouse and rat as well as many other mammalian species [31], and the main secondary structures (the alpa-helixes and the beta-chain strands) of the cavity are also conserved (Additional file 3: Figure S1). As inferred from the 3D structure prediction of the fusion protein, this cavity appeared to be exposed away from the sfGFP and in a favourable orientation for TCDD binding (Fig. 1b, Additional file 1: Table S1).

\section{Cloning of $A h R$ gene into the pRSET-sfGFP plasmid}

The AhR(LBD) was amplified from the cDNA of the HepG2 cell line using a couple of AhR-specific primers, and a single DNA fragment of $630 \mathrm{bp}$ was therefore obtained and cloned in the pRSET-sfGFP plasmid (Fig. 2a). After transformation in E. coli, positive colonies were selected and used for plasmid mini-preparation and the correct constructs were verified by $\mathrm{BamHl} / E c o$ RI-digestion (Fig. 2b). An expected band of 609 bp corresponding to the LBD fragment was only resulted from digesting the construct pRSET-sfGFP-AhR and not the original plasmid (Fig. 2b). Furthermore, the cloned insert in the pRSET-sfGFP-AhR was entirely sequenced and aligned to the human AhR gene sequence (Accession: NM_001621) to confirm the absence of mutations.

Plasmids like the pRSET with a $\mathrm{T}_{7}$ promoter are widely used for high throughput protein expression in E. coli strains carrying the lambda DE3 lysogen [32] (Additional file 4: Figure S2 and Additional file 2: Table S2).

\section{Expression of the fluorescent sfGFP-AhR fusion protein}

Expression of the sfGFP-AhR was carried out after transformation of E. coli BL21 (DE3) Gold cells with the confirmed pRSET-sfGFP-AhR construct. A remarkable expression of a $50 \mathrm{kDa}$ green $s f \mathrm{GFP}-\mathrm{AhR}$ fusion protein was observed after IPTG induction. Unexpectedly, the expressed sfGFP-AhR was mainly accumulated in the form of the inclusion bodies, and a small amount of the protein was foundsoluble in the cytoplasm (Fig. 3a). Fluorescence properties of the sfGFP-AhR fusion protein, recovered from the inclusion bodies by solubilizing in urea, were investigated using serial concentrations. Apparently, the $s f G F P-A h R$ exhibited a fluorescence, but to a lesser extent than the sfGFP, at a standard wavelength for excitation $(485 \mathrm{~nm})$ and emission $(538 \mathrm{~nm})$ (Fig. 3b). However, fluorescence spectra of the free and fusion sfGFP seemed to be identical at several pairs of wavelengths for excitation and emission and differ slightly from the spectrum of the fluorescein (Fig. 3b, inset). 


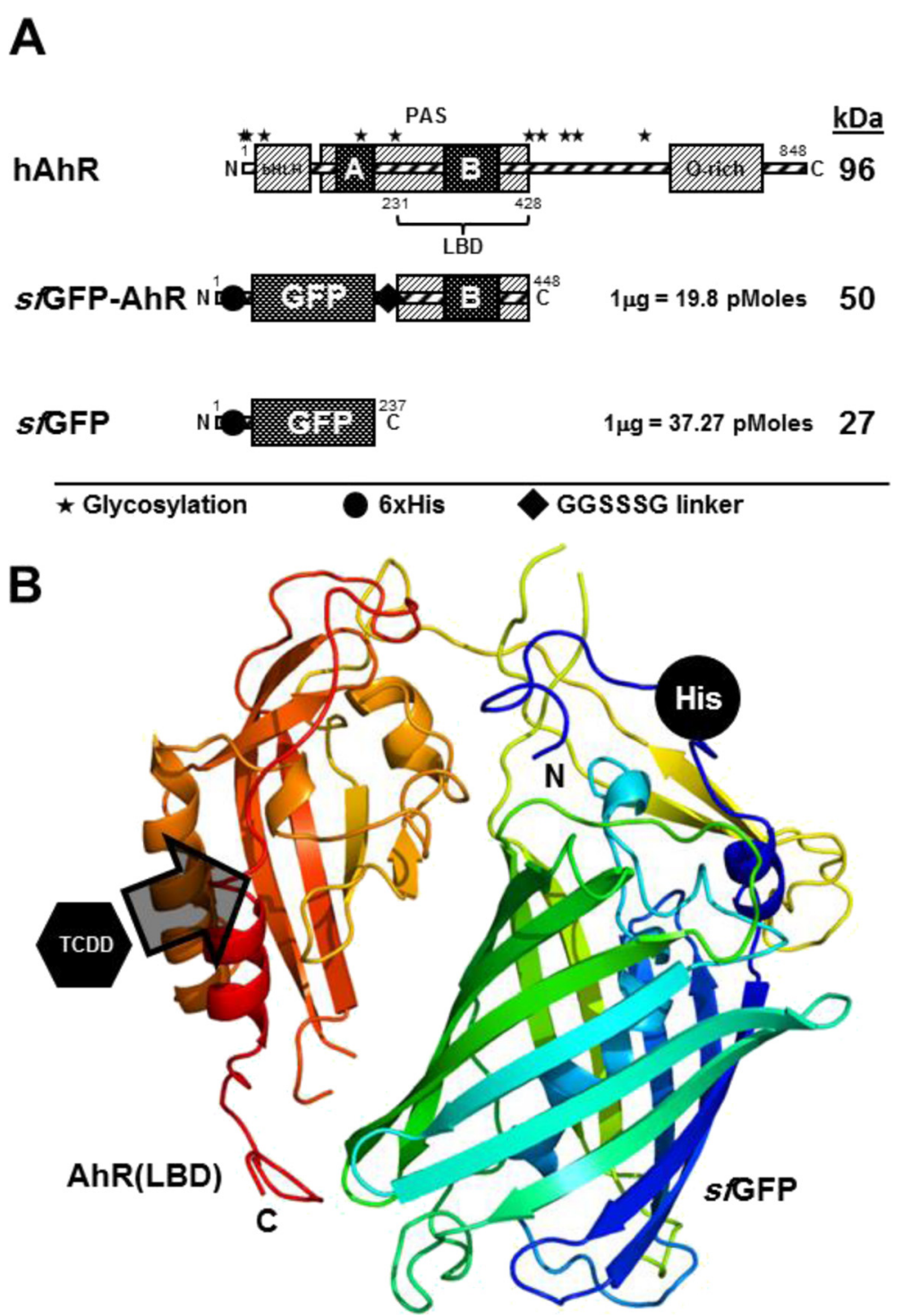

Fig. 1 Designing of the sfGFP-AhR fusion protein. a Schematic representation of the hAhR gene and the two recombinant proteins; sfGFP-AhR and sfGFP, used in this study. The different domains of the AhR gene are shown and the cloned LBD domain (231-428 aa) is indicated. The theoretical molecular size $(\mathrm{kDa})$ and molecular weight (pMoles $/ \mathrm{\mu g}$ ) are shown to the right of each recombinant construct. Positions of the different elements; $6 \times$ His tag, GGSSSG linker and glycosylation sites, are indicated using specific symbols $\bullet$, and *, respectively. $\mathbf{b}$ Cartoon representation of the modelled 3D structure of the sfGFP-AhR fusion, where TCDD binding cavity and the N-terminal $6 \times$ His tag are shown. Structure simulation was predicted using Phyre2 server [47]

\section{Optimization of in vitrosfGFP-AhR refolding}

Due to the insolubility of the expressed AhR-sfGFP fusion, an in vitro solubilizing and refolding process was required to obtain an active form of the protein. Several reagents were added into the buffer of the inclusion bodies to improve the solubility and prevent the aggregation of the sfGFP-AhR protein as described in Materials and Methods. By comparing the fluorescence in soluble and insoluble fractions, only three reagents $(0.4 \mathrm{M}$ Arginine, $0.1 \mathrm{M}$ Lauroylsarcosine and $0.1 \% \mathrm{SDS}$ ) were able to to solubilize the inclusion bodies completely, and no remaining fluorescence could be detected in the insoluble fraction (Fig. 4a). Other used reagents were not as effective as these three conditions in solubilizing the sfGFP-AhR. Next, serial concentrations of Arginine (from 0 to $0.4 \mathrm{M}$ ) and Lauroylsarcosine (from 0 to $0.08 \mathrm{M}$ ) were examined for optimizing the solubilization of $s f G F P-A h R$ inclusion bodies. As we did before, the fluorescence of the sfGFP-AhR was measured in soluble and insoluble fractions. Effectively, protein solubility was enhanced using increasing concentrations of both reagents (Fig. 4b and c). Consequently, $0.1 \mathrm{M}$ Arginine was 

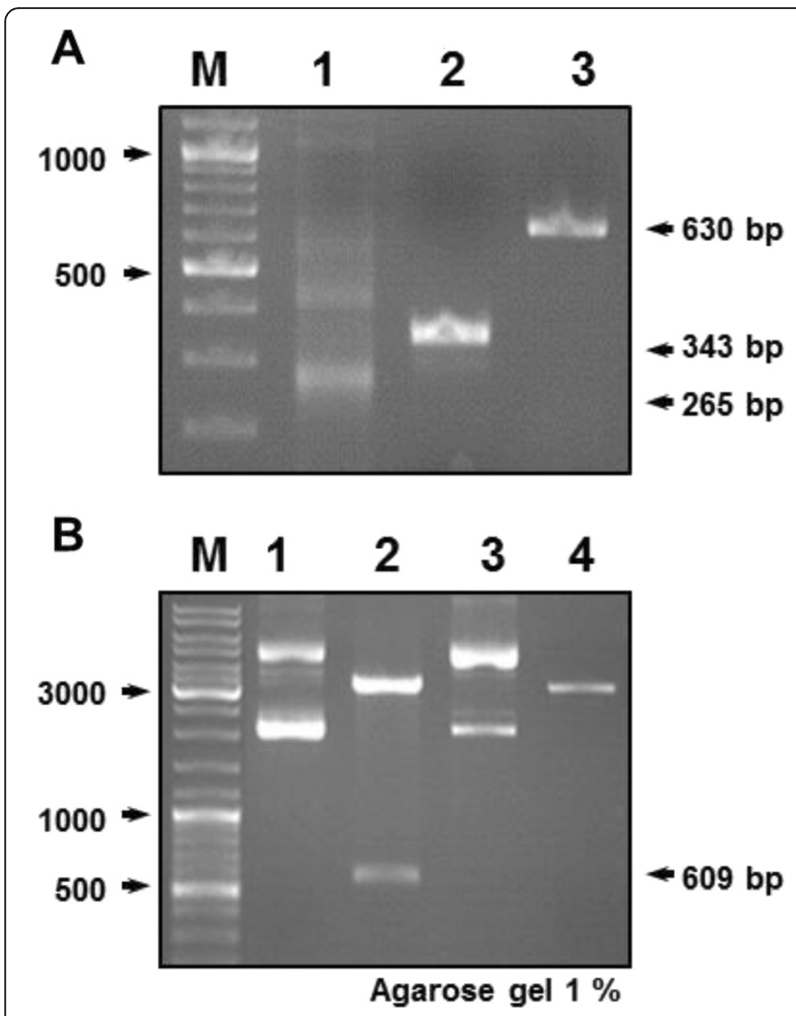

Fig. 2 Construction of the pRSET-sfGFP-AhR plasmid. a PCR amplification was performed on the CDNA of HepG2 cells and the products were separated on a $1 \%$ agarose gel; a fragment (265 bp) of actin gene as a control (lane 1), small domain from the AhR gene using internal primers (lane 2) and the full-length of LBD (lane 3). Expected sizes of the amplified bands are shown to the right. $\mathbf{b}$ $1 \%$ agarose gel containing the pRSET-sfGFP-AhR (lanes 1\&2) and pRSET-sfGFP (lanes 3\&4); undigested (lanes 1\&3) or digested with BamHI/EcoRl restriction enzymes (lanes 2\&4). The extracted AhR insert is indicated with an arrow to the right

used for refolding and solubilizing of the sfGFP-AhR fusion from the inclusion bodies.

\section{Purification and immunodetection of the SfGFP-AhR}

Purification of the sfGFP-AhR from the solubilized inclusion bodies was performed on an immobilized-metal affinity chromatography using Nickel-charged NTA column (Fig. 5a). The integrity of the purified proteins, the $s f G F P-A h R$ and $s f G F P$, was analysed by SDS-PAGE and immunoblotting by either mouse anti-GFP or anti$6 \times$ His antibodies. Two major specific bands of about 50 and $27 \mathrm{kDa}$, corresponding to the $s f \mathrm{GFP}-\mathrm{AhR}$ and $s f \mathrm{GFP}$, respectively, were immune-detected on the membranes (Fig. 5b). The purified $s f G F P-A h R$ and $s f G F P$ were tested in a solid-phase ELISA through targeting their GFP and $6 \times$ His tags using different monoclonal and polyclonal antibodies. Obviously, the GFP tag allowed much more efficient detection of both ELISA-immobilized proteins compared to the $6 \times$ His tag, and this could be related to the great differences in the size and epitopes availability

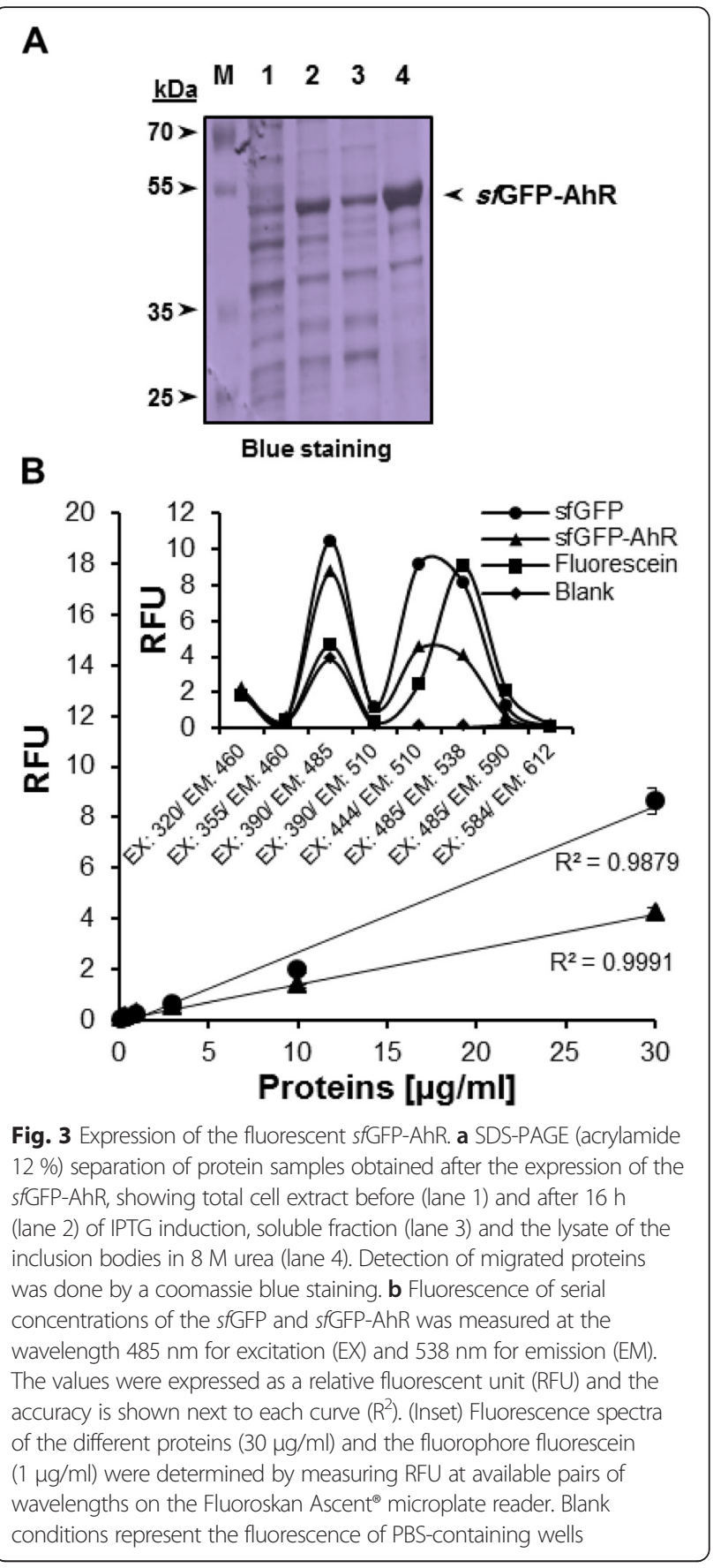

of these two tags (Fig. 5c). These data indicated clearly that the immunodetection of the sfGFP-AhR fusion could be achieved efficiently using specific anti-GFP antibodies.

Detection of the interaction of the SfGFP-AhR with dioxin The sfGFP-AhR was effectively able to detect the immobilized TCDD using either anti- $6 \times$ His or anti-GFP antibodies (Fig. 6a and b). However, the major absorbance at $450 \mathrm{~nm}$ (about 1.4) was detected in the case of anti-GFP, 


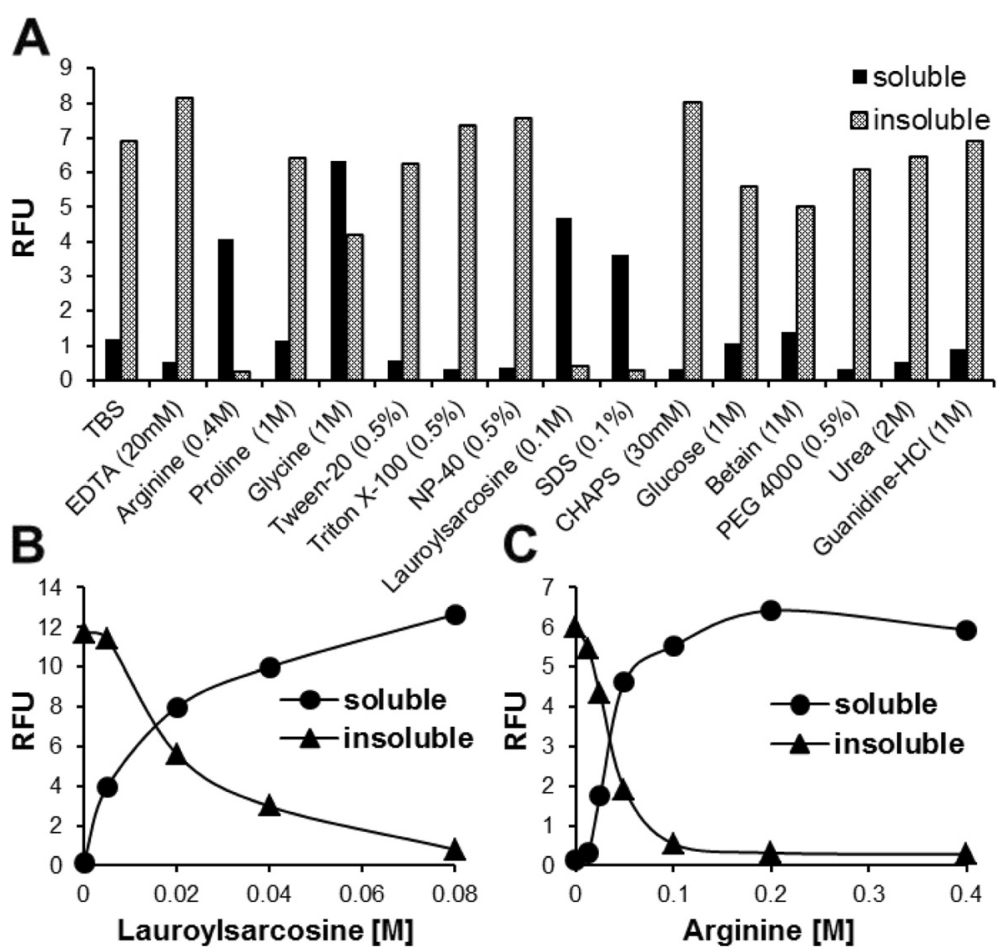

Fig. 4 Optimization of the in-vitro sfGFP-AhR refolding. a Several additives were added at their indicated optimal concentration to solubilize a pellet $(0.1 \mathrm{mg}$ in $1 \mathrm{ml}$ ) of sfGFP-AhR inclusion bodies (soluble fraction). Remaining insoluble precipitates were recovered by centrifugation and solubilized in $8 \mathrm{M}$ urea (insoluble fraction). Serial molar concentrations of Lauroylsarcosine (b) and Arginine (c) used for solubilizing the pellet of sfGFP-AhR inclusion bodies. The RFU was measured (EX: 485/EM: 538) in soluble and insoluble fractions for each additive and for the different concentrations of the last two additives

and the $s f$ GFP was used as a negative control (Fig. 6b). As a positive control, the immobilized TCDD gave an absorbance of 0.8 when detected using specific antiTCDD (a-TCDD) antibody. Competitive ELISA is usually used as an analytical method to quantify dioxins in food, feed and environmental soil samples. The principle of the competitive ELISA is that free dioxins in the samples compete with the immobilized dioxin for binding to the detecting molecule (sfGFP-AhR or a-TCDD). Therefore, we used this system of detection to confirm the sensitivity of the sfGFP-AhR fusion towards TCDD. For this purpose, we firstly determined the optimal effective concentration of the sfGFP-AhR necessary for achieving $75 \%$ of the maximal detection of the immobilized TCDD $\left(E^{75}=n M\right)$. To do that, serial logarithmic molar concentrations of the $s f G F P-A h R$ were incubated in the wells of TCDD-pre-coated microplate then the binding activity was evaluated using a rabbit-anti-GFP antibody. A linear correlation between the absorbance and the concentrations of the $s f G F P-A h R$ was clearly observed, and $\mathrm{EC}^{75}$ of the detection was estimated to be about $33.1 \pm 9.2 \mathrm{nM}$ (Fig. 7a). Based on this data, a sfGFP-AHR concentration of $30 \mathrm{nM}$ was subsequently used. Different concentrations of TCDD (in ppt) were incubated with either the $s f G F P-A h R$ or a-TCDD, and then added into the wells of the TCDD-pre-coated microplate. Binding of the detecting molecule to immobilized TCDD was finally revealed by the secondary antibodies. Absorbance values were inversely proportioned to the amount of free TCDD in the samples. In the case ofthea-TCDD, $\mathrm{EC}^{50}$ of the detection was $11.9 \pm 0.4 \mathrm{ppt}$ and the detection range (from $\mathrm{EC}^{99}$ to $\mathrm{EC}^{1}$ ) was from $1.1 \pm 0.05 \mathrm{ppt}$ to $83.7 \pm$ $2.6 \mathrm{ppt}$, whereas the $s f \mathrm{GFP}-\mathrm{AhR}$ showed a higher $\mathrm{EC}^{50}$ of about $346.5 \pm 21.4$ ppt with a wider detection range from $0.8 \pm 0.04$ ppt to $51600 \pm 15500$ ppt (Fig. $7 b$ ). In conclusion, the sum of dioxin in the target samples could be detected using a similar method and its exact concentration could be calculated by a comparison with the same standard curve and logarithmic fit equation.

\section{Discussion}

To date, many reports have described the heterologous expression of the recombinant AhR in both prokaryotic and eukaryotic cell types. Particularly, the recombinant expression of the ligand-binding competent AhR would have significant advantages for many bioanalytical applications regarding dioxins detection. The majority of the described methods take advantage of the ability of dioxin/AhR complexes to trigger certain signal transduction pathways [33]. Transgenic tobacco plants carrying 


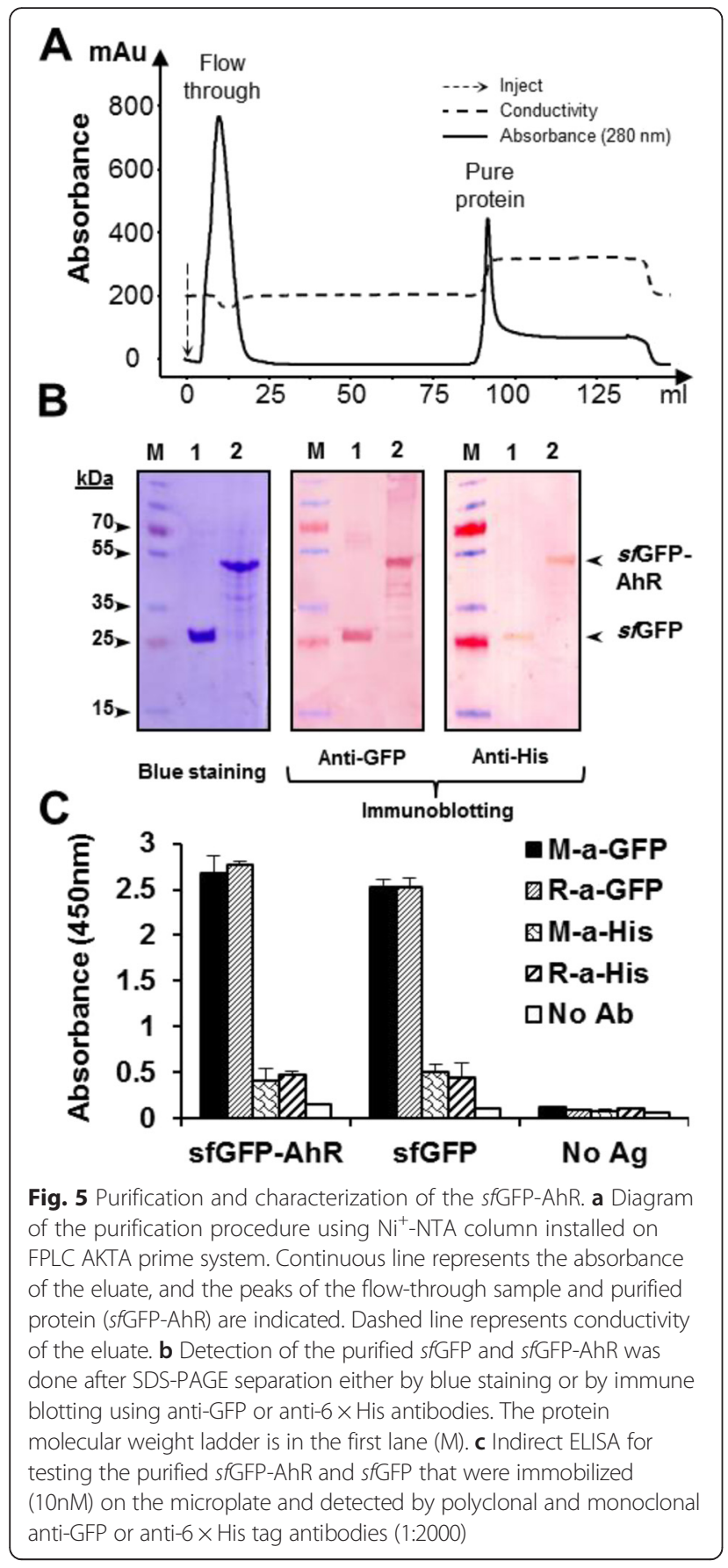

the recombinant mouse AhR-mediated $\beta$-glucuronidase (GUS) reporter gene expression system was designed and showed a significantly increased GUS activity when treated with certain AhR ligands [34]. Such assays did not need any extraction and purification of chemicals and seemed to be useful for a biochemical assay of dioxins and dioxin-like compounds [35]. Furthermore, recombinant yeast cells Saccharomyces cerevisiae have been designed as a dioxin bioassay system, in which the expressed full length human or mouse AhR/Arnt complexes form after

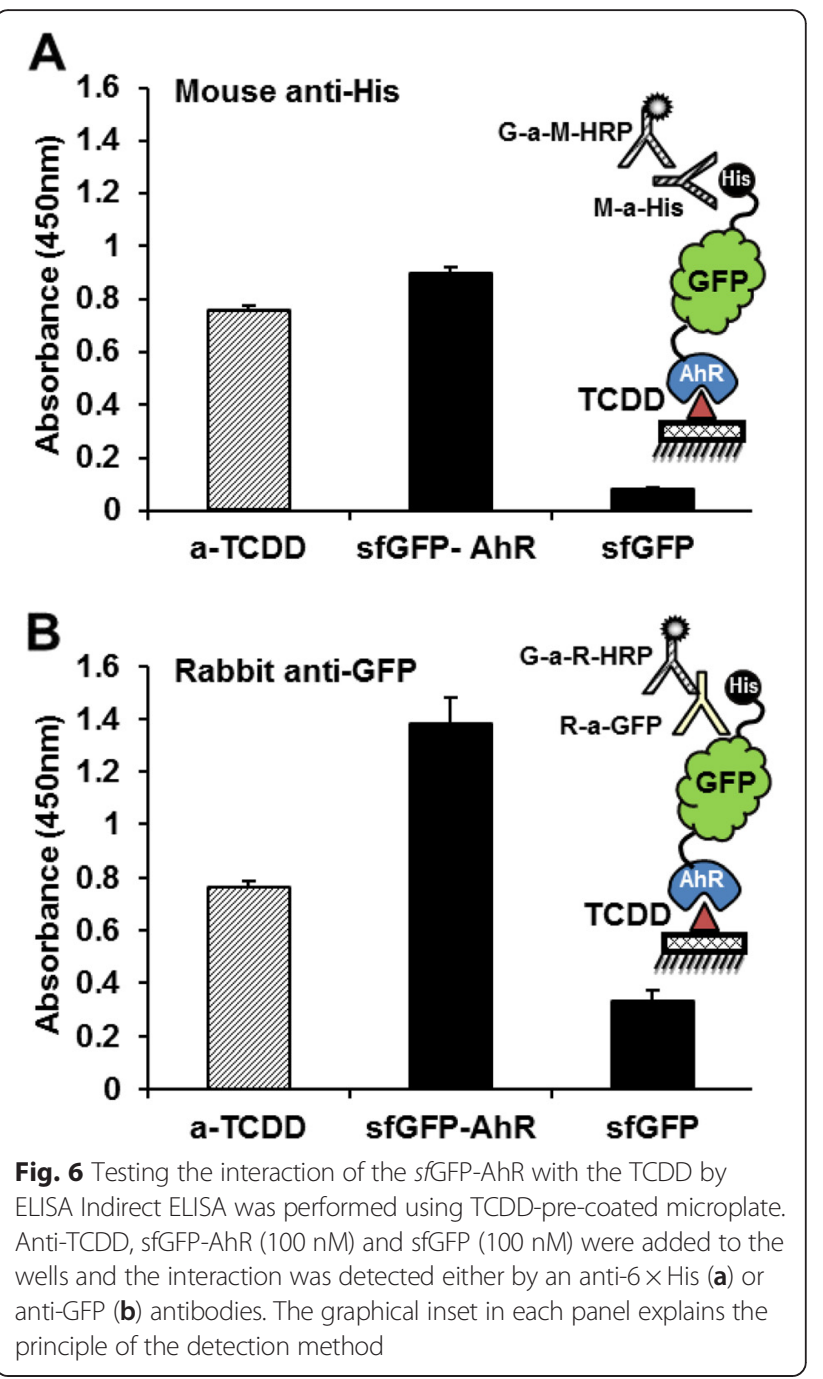

ligand binding and interact with a responsive element located on the promoter of $B$-galactosidase reporter gene [36], and this system was utilized to identify potent endogenous AhR ligands from human urine and bovine serum [37]. Baculovirus expression vector system was utilized for high level production of the full-length human and rat AhR in Spodopterafrugiperda (Sf9), and the recombinant proteins were active in the quantitative analysis of the ligand [38]. In fact, the absence of the disulfide bridges and glycosylation sites in the LBD of AhR makes it a more suitable target for expression in E. coli. However, several attempts for LBD expression in $E$. coli were unsuccessful because of its instability and its high tendency to aggregate because of misfolding or incomplete folding processes [23]. Therefore, fusion protein technology is considered one of the best solutions to enhance the protein expression and solubility. For example, it chaperones the proper folding, facilitates the purification and reduces the protein degradation or toxic effects [39]. Many fusion moieties are available to improve the expression and 


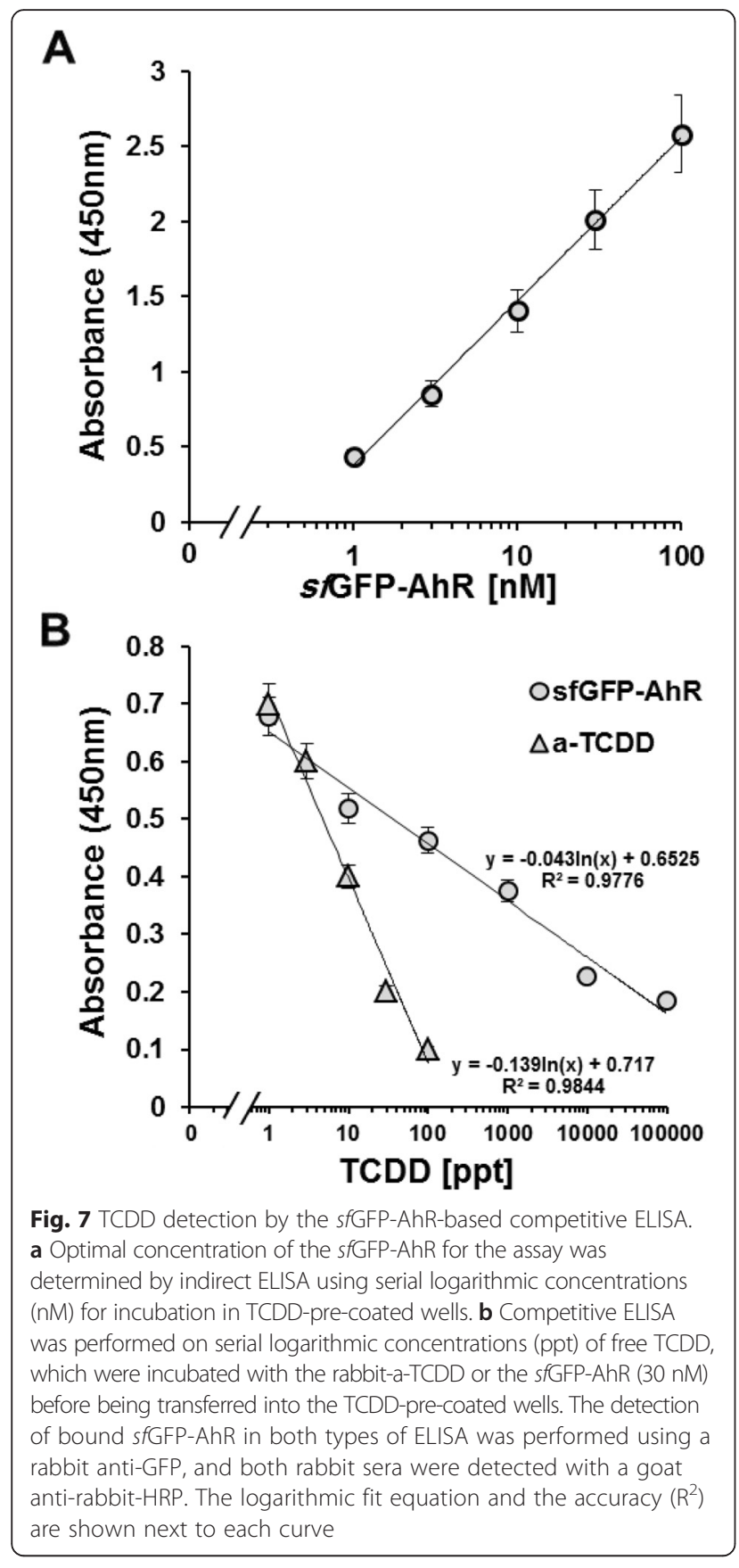

purification of proteins, including glutathione S-transferase (GST), maltose-binding protein (MBP), thioredoxin A (TrxA), and the GFP [40].

In the current study, we successfully produced the LBD of AhR in a fusion with the $s f \mathrm{GFP}$, resulting in its expression at a high level ( $150 \mathrm{mg} / \mathrm{L}$ of bacteria culture $)$ in the cytoplasm of E. coli. Despite its well documented stability and solubility, the enhanced form of the GFP, the superfolder GFP, failed to maintain the correct folding of the expressed AhR, leading therefore to its accumulation into the inclusion bodies. This was disappointing since many reports described the recovery of several insoluble proteins from the inclusion bodies simply by their merging with the sfGFP, e.g. the human growth hormone [41], and in certain cases, this resulted in the enhancement of their physiological roles [42]. The culture and induction conditions, including temperatures and IPTG concentrations, may play a critical role in the solubility of many recombinant proteins, especially, when some stringent culture conditions provoke the production of large quantities of the exogenous protein that the cell folding machinery fails to process [43]. Hence, several conditions were tested to assess their influence on the solubility of the sfGFP-AhR which was simply monitored by comparing the colour of the bacterial lysate and precipitate. Unfortunately, the fusion greenish protein was always found in the inclusion bodies regardless of the conditions used, even with the mild ones; Tm $10{ }^{\circ} \mathrm{C}$ and $0.1 \mathrm{mM}$ IPTG, on the contrary, free $s f G F P$ was always soluble when expressed from the plasmid pRSET-sfGFP, using many different culture conditions (data not shown).

Arginine has long been used as an interesting additive for solubilizing the inclusion bodies and enhancing protein solubility and stability [44]. Its mechanism of action relies on inhibiting the aggregation of misfolded proteins and surrounding their exposed hydrophobic surfaces. Furthermore, 0.5-2MArginine, can be used to actively extract the folded proteins from insoluble pellets obtained after lysing E. coli cells [44]. Comparing several tested solubilizing reagents reported in the literature [29], we found that low concentrations of Arginine, e.g. $0.1 \mathrm{M}$, were enough to refold totally $s f \mathrm{GFP}-\mathrm{AhR}$ from the inclusion bodies, and even without a prior step for solubilizing the inclusion bodies in $6 \mathrm{M}$ guanidine- $\mathrm{HCl}$ or $8 \mathrm{M}$ urea. Lauroylsarcosine showed a similar effect on the inclusion bodies of the $s f G F P-A h R$, however its severity, as a strong ionic detergent, compared to the Arginine which is barely a stabilizing amino acid, makes its uses unfavourable in the production procedure of the $s f G F P-A h R$. Nevertheless, we speculated from this experiment that the structure of the sfGFP-AhR is moderately misfolded, making its recovery from the inclusion bodies an easy and inexpensive task to perform. This could be related to the sfGFP owing to the folding reputation it has, beside, its visible and florescent signals which made it possible to track the transformation of the fusion protein between the insoluble and soluble states, especially when different solubilizing additives were used. Alternatively, the aggregation problem of the AhR can be probably overcome by using eukaryotic heterologous expression systems such as animal or plant cells.

The ability ofsfGFP-AhR to bind to TCDD was proven by a standard and a competitive ELISA. This last type of ELISA is mainly used for the detection of very-small antigens that are difficult to be immobilized on a solid 
support, hard to purify and composed of a few number of epitopes, e.g. the haptens [14]. It is usually performed to quantify TCDD contamination in water, soil and sediment samples [12]. Despite the fact that anti-TCDD antibody showed more sensitivity for TCDD in competitive ELISA than the sfGFP-AhR, the detection range of the $s f G F P-A h R$ was much wider. According to the USAEnvironmental Protection Administration (EPA), the maximum limits for dioxins in wastewater are set to be $5 \mathrm{pg}$ I-TEQ per liter for new companies and $10 \mathrm{pg}$ I-TEQ per liter for existing companies. These ranges which equal 5 to $10 \mathrm{ppt}$ of total dioxins are being covered by estimated sensitivity range of the proposed sfGFP-AhR detection system. For enhancing the affinity, replacing the LBD in the sfGFP-AhR fusion, originated from human AhR, with the equivalent domain from mouse or rat $A h R$ can be suggested, because they are known to have better binding kinetics towards TCDD [38]. Nevertheless, this sfGFP-AhR-based immunoassay can be exploited as a method for analyzing large number of samples, from different sources, for contamination with TCDD, or any similar compounds. An estimated value of TCDD content can be attributed to the measured samples simply by comparing the logarithmic curve fit of the known concentrations of standard TCDD. Samples that are determined to be positive in this immunoassay can undergo congener-specific analyses by HR-GC/MS. Beside its analytical uses, the fluorescent sfGFP-AhR fusion can be used as a powerful method for bio-tracing of TCDD within the cells or tissues in diverse unexploited areas of research. For example, this bioanalytical method will surely contribute to a better understanding of the plant/dioxin relationship and therefore in deciphering the mechanisms involved in plant responses to dioxin exposure $[45,46]$.

\section{Conclusions}

In conclusion, dioxin binding domain of the AhR was produced in a fusion with an enhanced form of the GFP using an efficient E. coli protein expression system. After Arginine-refolding and affinity purification, the sfGFP-AhR fusion protein was able to bind to TCDD using a standard and competitive ELISA, and the detection limit of free TCDD in samples could reach very low levels ( 10 ppt).

\section{Additional files}

Additional file 1: Table S1. The primers used for the amplification and cloning of the AhR. The different parameters (application, name, length and sequence) of the primers used for $A h R$ gene amplification and cloning into the pRSET-sfGFP plasmid. (DOC $79 \mathrm{~kb}$ )

Additional file 2: Table S2. The different additives used for refolding the sfGFP-AhR. The basic information of the different additives used for solubilizing the sfGFP-AhR from the inclusion bodies. (DOC $95 \mathrm{~kb}$ )
Additional file 3: Figure S1. Alignment of the AhR(LBD) amino acids sequences. Amino acids sequence alignment of the $A h R(L B D)$ from human (hAhR, NP_001612), mouse (mAhR, NP_038492) and rat (rAhR, NP_037281). Similarity bar above the sequences and the different secondary structures (as attributed by Geneious software) are indicated with small arrows. The TCDD binding cavity and the PAS-B domains are indicated through which small boxes under the sequence refer to the amino acids whose side chains are implicated in the structure of the cavity (light coloured for lateral and dark for internal positions), as described before [31]. (DOC $272 \mathrm{~kb}$ )

Additional file 4: Figure S2. The structure of the plasmid pRSET-sfGFPAhR. Map of the plasmid construct pRSET-sfGFP-AhR, in which the inserted AhR (LBD) is indicated. The most important elements of the plasmid are shown, including the $\mathrm{T}_{7}$ promoter, $\mathrm{N}$-terminal $6 \times \mathrm{His}$ tag, two restriction sites (BamHI/EcoRI) used for insert ligation, ampicillin resistance gene (Amp), f1/PUC origin of replication and the sfGFP gene. (DOC $266 \mathrm{~kb}$ )

\section{Abbreviations}

AhR, aryl hydrocarbon receptor; bHLH/PAS, basic helix-loop-helix/Per-ARNTSim; GFP, superfolder green fluorescent protein; LBD, ligand binding domain; PCDDs, polychlorinated dibenzo-p-dioxins; PCDFs, polychlorinated dibenzofurans; TCDD, 2,3,7,8-Tetrachlorodibenzo-p-dioxin sf.

\section{Acknowledgments}

The authors would like to thank the Director General of the Atomic Energy Commission of Syria and the head of the Molecular Biology and Biotechnology department for their continuous support throughout this work.

\section{Funding}

This work was performed without any external funding.

\section{Availability of supporting Data}

The data set(s) supporting the results of this article is(are) included within the article (and its additional file(s)).

\section{Authors' contributions}

WF, carried out all experimental work. AH, designed TCDD-affinity experiments, participated in writing the manuscript. MMK, read and commented the manuscript. AA, led the work, designed all experiments, and wrote the manuscript. All authors read and approved the final manuscript.

\section{Competing interests}

The authors declare that they have no competing interests.

\section{Consent for publication}

"Not applicable".

Ethics approval and consent to participate

"Not applicable".

\section{Author details}

${ }^{1}$ Department of Animal Biology, Faculty of Sciences, Damascus University, Damascus, Syria. ${ }^{2}$ Division of Toxicology, Department of Molecular Biology and Biotechnology, Atomic Energy Commission of Syria (AECS), Damascus, Syria. ${ }^{3}$ Division of Microbiology and Immunology, Department of Molecular Biology and Biotechnology, Atomic Energy Commission of Syria (AECS), Damascus, Syria.

Received: 7 April 2016 Accepted: 13 June 2016

Published online: 21 June 2016

\section{References}

1. WHO: World Health Organization, Executive Summary on the Assessment of the health risk of dioxins, WHO Consultation, May 25-29 1998. www.who. int/pcs/docs/dioxin-exec-sum-final. 2016.

2. Huuskonen $\mathrm{H}$, Unkila M, Pohjanvirta $\mathrm{R}$, Tuomisto J. Developmental toxicity of 2,3,7,8-tetrachlorodibenzo-p-dioxin (TCDD) in the most TCDD-resistant and -susceptible rat strains. Toxicol Appl Pharmacol. 1994;124(2):174-80. 
3. Baker TK, Kwiatkowski AP, Madhukar BV, Klaunig JE. Inhibition of gap junctional intercellular communication by 2,3,7,8-tetrachlorodibenzo-pdioxin (TCDD) in rat hepatocytes. Carcinogenesis. 1995;16(10):2321-6.

4. Tuppurainen K, Asikainen A, Ruokojarvi P, Ruuskanen J. Perspectives on the formation of polychlorinated dibenzo-p-dioxins and dibenzofurans during municipal solid waste (MSW) incineration and other combustion processes. Acc Chem Res. 2003;36(9):652-8.

5. Meharg AA, Osborn D. Dioxins released from chemical accidents. Nature 1995;375(6530):353-4.

6. WHO: World Health Organization Fact Sheet No 225, Dioxins and Their Effects on Human Health, June 1999, http://www.who.int/mediacentre/ factsheets/fs225/en/. 2016.

7. Pollitt F. Polychlorinated dibenzodioxins and polychlorinated dibenzofurans. Regul Toxicol Pharmacol. 1999;30:63-8.

8. McConkey DJ P, Hartzell SK, Duddy H, Hakansson S. Orrenius: 2,3,7,8 Tetrachlorodibenzo-p-dioxin kills immature thymocytes by $\mathrm{Ca}^{2+}$-mediated endonuclease activation. Science. 1988;242(4876):256-9.

9. Carney SA, Prasch AL, Heideman W, Peterson RE. Understanding dioxin developmental toxicity using the zebrafish model. Birth Defects Res Part A Clin Mol Teratol. 2006;76(1):7-18.

10. Toth K, Somfai-Relle S, Sugar J, Bence J. Carcinogenicity testing of herbicide 2,4,5-trichlorophenoxyethanol containing dioxin and of pure dioxin in Swiss mice. Nature. 1979;278(5704):548-9.

11. Caserini S, Cernuschi S, Giugliano M, Grosso M, Lonati G, Mattaini P. Air and soil dioxin levels at three sites in Italy in proximity to MSW incineration plants. Chemosphere. 2004;54(9):1279-87.

12. Hanano A, Ammouneh H, Almousally I, Alorr A, Shaban M, Alnaser AA, Ghanem I. Traceability of polychlorinated dibenzo-dioxins/furans pollutants in soil and their ecotoxicological effects on genetics, functions and composition of bacterial community. Chemosphere. 2014;108:326-33.

13. Tian W, Xie HQ, Fu H, Pei X, Zhao B. Immunoanalysis methods for the detection of dioxins and related chemicals. Sensors. 2012;12(12):16710-31.

14. Okuyama M, Kobayashi N, Takeda W, Anjo T, Matsuki Y, Goto J, Kambegawa A, Hori S. Enzyme-linked immunosorbent assay for monitoring toxic dioxin congeners in milk based on a newly generated monoclonal anti-dioxin antibody. Anal Chem. 2004;76(7):1948-56.

15. Eichbaum K, Brinkmann M, Buchinger S, Reifferscheid G, Hecker M, Giesy JP, Engwall $M$, van Bavel $B$, Hollert $H$. In vitro bioassays for detecting dioxin-like activity-application potentials and limits of detection, a review. Sci Total Environ. 2014:487:37-48

16. Hahn ME. Aryl hydrocarbon receptors: diversity and evolution. Chem Biol Interact. 2002;141(1-2):131-60.

17. Okey AB, Franc MA, Moffat ID, Tijet N, Boutros PC, Korkalainen M, Tuomisto J, Pohjanvirta R. Toxicological implications of polymorphisms in receptors for xenobiotic chemicals: the case of the aryl hydrocarbon receptor. Toxicol Appl Pharmacol. 2005;207:43-51.

18. Beischlag TV, Luis Morales J, Hollingshead BD, Perdew GH. The aryl hydrocarbon receptor complex and the control of gene expression. Crit Rev Eukaryot Gene Expr. 2008;18:207-50.

19. Kazlauskas A, Poellinger L, Pongratz I. Evidence that the co-chaperone p23 regulates ligand responsiveness of the dioxin (Aryl hydrocarbon) receptor. J Biol Chem. 1999;274:13519-24.

20. Reyes $H$, Reisz-Porszasz S, Hankinson O. Identification of the Ah receptor nuclear translocator protein (Arnt) as a component of the DNA binding form of the Ah receptor. Science. 1992;256:1193-5.

21. Kewley RJ, Whitelaw ML, Chapman-Smith A. The mammalian basic helixloop-helix/PAS family of transcriptional regulators. Int J Biochem Cell Biol. 2004;36(2):189-204.

22. Fukunaga BN, Probst MR, Reisz-Porszasz S, Hankinson O. Identification of functional domains of the aryl hydrocarbon receptor. J Biol Chem. 1995; 270(49):29270-8

23. Coumailleau P, Poellinger L, Gustafsson JA, Whitelaw ML. Definition of a minimal domain of the dioxin receptor that is associated with $\mathrm{Hsp90}$ and maintains wild type ligand binding affinity and specificity. J Biol Chem. 1995;270(42):25291-300.

24. Lee $\mathrm{CH}$. Recombinant Green Fluorescent Protein Derivatives as a Fusion Tag for in vitro Experiments. Interdiscip Bio Central. 2009;1(1):1-15.

25. Chalfie M, Tu Y, Euskirchen G, Ward WW, Prasher DC. Green fluorescent protein as a marker for gene expression. Science. 1994;263(5148):802-5.

26. Gerdes HH, Kaether C. Green fluorescent protein: applications in cell biology. FEBS Lett. 1996;389(1):44-7.
27. Pedelacq JD, Cabantous S, Tran T, Terwilliger TC, Waldo GS. Engineering and characterization of a superfolder green fluorescent protein. Nat Biotechnol. 2006;24(1):79-88.

28. Al-Homsi L, Al-Assad JM, Kweider M, Al-Okla S, Abbady AQ. Construction of pRSET-sfGFP plasmid for fusion-protein expression, purification and detection. Jordan J Biol Sci. 2012;5(4):279-88.

29. Alibolandi M, Mirzahoseini H. Chemical assistance in refolding of bacterial inclusion bodies. Biochem Res Int. 2011;2011:631607.

30. Twair A, Al-Okla S, Zarkawi M, Abbady AQ. Characterization of camel nanobodies specific for superfolder GFP fusion proteins. Mol Biol Rep. 2014; 41(10):6887-98.

31. Pandini A, Soshilov AA, Song Y, Zhao J, Bonati L, Denison MS. Detection of the TCDD binding-fingerprint within the Ah receptor ligand binding domain by structurally driven mutagenesis and functional analysis. Biochemistry. 2009:48(25):5972-83.

32. Studier FW, Rosenberg AH, Dunn JJ, Dubendorff JW. Use of T7 RNA polymerase to direct expression of cloned genes. Methods Enzymol. 1990;185:60-89.

33. Takigami H, Mitsuhara Y, Matsuyama K, Sakai S. Comparison of DR-CALUX to HRGC/HRMS - TEQ monitoring during Kanechlor PCB degradation process using metallic sodium dispersion. Organohalogen Compd. 2004;66:637-40.

34. Inui H, Gion K, Utani Y, Wakai T, Kodama S, Eun H, Kim YS, Ohkawa H. Assays of dioxins and dioxin-like compounds in actually contaminated soils using transgenic tobacco plants carrying a recombinant mouse aryl hydrocarbon receptor-mediated beta-glucuronidase reporter gene expression system. J Environ Sci Health B. 2012;47(1):59-65.

35. Shimazu S, Ohta M, Ohkawa $H$, Ashida H. Assays of polychlorinated biphenyl congeners and co-contaminated heavy metals in the transgenic Arabidopsis plants carrying the recombinant guinea pig aryl hydrocarbon receptor-mediated beta-glucuronidase reporter gene expression system. J Environ Sci Health B. 2012;47(10):925-32.

36. Kawanishi M, Sakamoto M, Ito A, Kishi K, Yagi T. Construction of reporter yeasts for mouse aryl hydrocarbon receptor ligand activity. Mutat Res. 2003 540(1):99-105.

37. Adachi J, Mori Y, Matsui S, Takigami H, Fujino J, Kitagawa H, Miller 3rd CA Kato T, Saeki K, Matsuda T. Indirubin and indigo are potent aryl hydrocarbon receptor ligands present in human urine. J Biol Chem. 2001; 276(34):31475-8.

38. Fan MQ, Bell AR, Bell DR, Clode $S$, Fernandes A, Foster PM, Fry JR, Jiang $T$, Loizou G, MacNicoll A, et al. Recombinant expression of aryl hydrocarbon receptor for quantitative ligand-binding analysis. Anal Biochem. 2009;384(2): 279-87.

39. Davis GD, Elisee C, Newham DM, Harrison RG. New fusion protein systems designed to give soluble expression in Escherichia coli. Biotechnol Bioeng 1999:65(4):382-8.

40. Hall JP. Applying Fusion Protein Technology to E. coli. BioPharm Int. 2007; 2007:1-11.

41. Abbady AQ, Al-Shemali R, Mir Assaad J, Murad H. Generation and characterization of nanobodies against rhGH expressed as sfGFP fusion protein. Gen Comp Endocrinol. 2014;204:33-42.

42. Tropea JE, Cherry S, Waugh DS. Expression and purification of soluble His(6)-tagged TEV protease. Methods Mol Biol. 2009;498:297-307.

43. Yamaguchi $H$, Miyazaki M. Refolding techniques for recovering biologically active recombinant proteins from inclusion bodies. Biomolecules. 2014;4(1):235-51.

44. Tsumoto K, Umetsu M, Kumagai I, Ejima D, Philo JS, Arakawa T. Role of arginine in protein refolding, solubilization, and purification. Biotechnol Prog. 2004;20(5):1301-8.

45. Hanano A, Almousally I, Shaban M. Phytotoxicity effects and biological responses of Arabidopsis thaliana to 2,3,7,8-tetrachlorinated dibenzo-pdioxin exposure. Chemosphere. 2014;104:76-84.

46. Hanano A, Almousally I, Shaban M, Moursel N, Shahadeh A, Alhajji E. Differential tissue accumulation of 2,3,7,8-Tetrachlorinated dibenzo-p-dioxin in Arabidopsis thaliana affects plant chronology, lipid metabolism and seed yield. BMC Plant Biol. 2015;15:193. DOI:10.1186/s12870-015-0583-5.

47. Kelley LA, Mezulis S, Yates CM, Wass MN, Sternberg MJ. The Phyre2 web portal for protein modeling, prediction and analysis. Nat Protoc. 2015;10(6):845-58. 